$17^{\text {th }}$ International Congress of Metrology, 02001 (2015)

DOI: $10.1051 /$ metrology / 201502001

(C) Owned by the authors, published by EDP Sciences, 2015

\title{
Numerical simulations: will they become mandatory for better management of measurement process?
}

\author{
Christian Rageade ${ }^{1}$, Alexandra Werlé ${ }^{1}$, Imen Canova ${ }^{1}$, Carole Tourneur ${ }^{1}$ and Anne Duraffourg ${ }^{1}$ \\ ${ }^{1}$ BioMérieux, Chemin de l'Orme, 69290 Marcy L'Etoile, France
}

\begin{abstract}
Résumé. Les normes ISO 17511 [1] et ISO18189 [2] demandent aux fournisseurs de méthode de diagnostic in vitro d'établir les incertitudes des étalons destinés aux laboratoires de biologie médicale. Dans ce document, nous nous proposons de montrer comment l'utilisation des méthodes de Monte Carlo pour calculer l'incertitude d'un étalon, peut conduire à développer un véritable simulateur d'un processus industriel complexe. Il s'agit du processus d'un système d'analyse semi automatisé pour le dosage des immunoessais: le Vidas ${ }^{\circledR}$ produit par bioMérieux. A la fin de la simulation, le nombre de résultats obtenus par simulation est comparable à la quantité de résultats réellement obtenue sur le terrain pendant le cycle de vie du produit. Ce simulateur modélise à la fois la réaction immunoenzymatique en fonction des variables physiques, mais aussi tout le processus de raccordement métrologique du dosage de l'étalon international jusqu'aux résultats du laboratoire. Pour alimenter ce simulateur, les données expérimentales provenant de plans d'expériences réalisés pendant le développement des produits sont utilisées. L'implémentation de ce simulateur offre de nouvelles possibilités pour l'établissement des performances de nos produits. Cet instrument virtuel a été validé sur 50 produits, il commence à être utilisé pour développer les nouveaux dosages. Des gains sont attendus sur les délais de développement et sur l'amélioration des ratios coût-qualité.
\end{abstract}

\section{Introduction}

Medical laboratories use commercialized kits provided by In-Vitro Diagnostics (IVD) manufacturers in order to provide clinicians and patients quantitative assay results. Calibrators with assigned concentrations are often included in such assay kits, for which IVD manufacturers need to provide associated uncertainty (as described in ISO 17511 [1]). The knowledge of this uncertainty is very important for the medical laboratories, in order for them to establish the uncertainty of the assay result provided to the clinician (as described in ISO18189 [2]). For its immunoassays products range, bioMerieux's objective was to establish uncertainty of the calibrators for about fifty quantitative assay kits. In order to facilitate and automate these calculations, a global model was developed to enable calculations of uncertainties based on simulations.

In this article, the development of the model will be explained, as well as the new opportunities for applications that were investigated.

\section{Industrial context}

\subsection{Presentation of the system}

bioMerieux offers a system, Vidas ${ }^{\circledR}$, that can be used in association with assay kits to perform immunology-based diagnostics. Around a hundred such kits are available, among them 50 or so allowing quantitative measurement of steroids, hormones, antibodies, tumorous markers, etc. in low concentrations. An assay kit typically contains some reagents, a calibrator and some controls.
The immune-enzymatic reaction happens inside of the instruments, lasting 15 to 30 minutes depending on the assay. A fluorescence signal is then emitted by the instrument, which is proportional to the input quantity of analyte in the tested sample.

\subsection{Immuno-enzymatic reaction and calibration}

In order to deliver results expressed in concentration, the mathematical relationship between the measured raw fluorescence signal and concentrations needs to be established using calibrators with known predefined titers. Since this relationship is not linear, several calibrators at different concentration levels need to be tested in replicate. Typically the model uses a fourthorder polynomial regression model (Figure 1).

Figure 1: Typical calibration curve. 


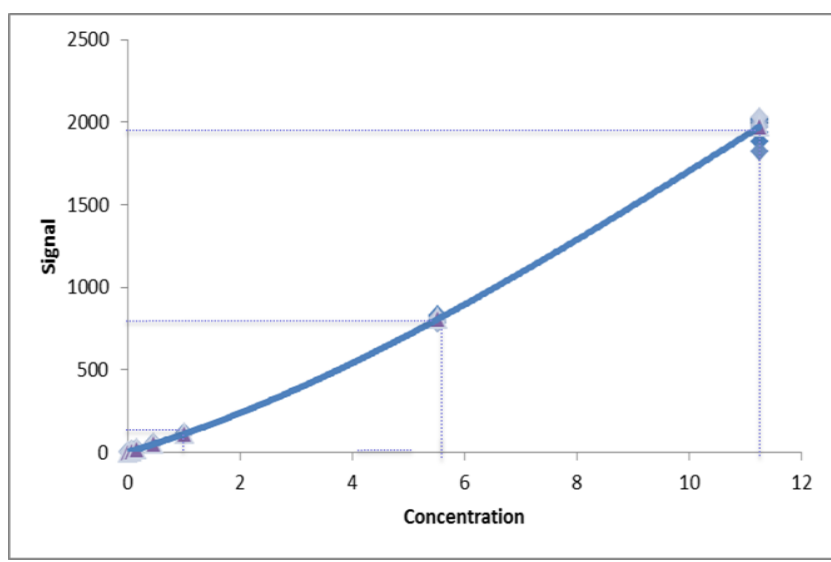

\subsection{Metrological traceability}

In order to express concentration results in standardized units (e.g. International units), metrological traceability is a key process during the development of a new assay. It consists of a hierarchical chain based on 3 types of calibrators: an international calibrator, a manufacturer calibrator and a medical laboratory calibrator (Figure 2).

Figure 2: Metrological traceability

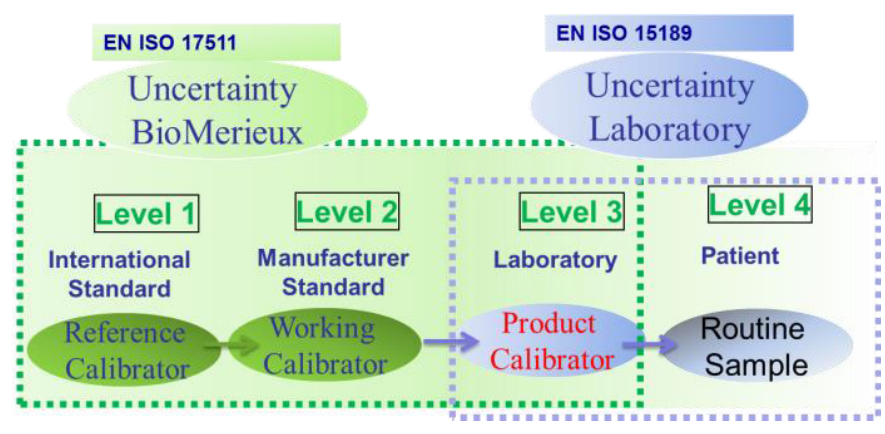

\subsection{Production and routine use}

For each batch of reagent produced by bioMerieux, the calibration curve is established during the production process. Then, the medical laboratory periodically uses the calibrator included in the Vidas kits to adjust the preestablished calibration curve. This account for instrument variability or drift in time.
The reagents have a limited shelf life (around 18 months typically), so several batches of reagents of the same assay can be used within a laboratory on one or several instruments, depending on their needs. This can lead to different variability across the laboratories.

\section{Development of the simulator}

\subsection{Strategy}

In order to assess the uncertainty of the concentration of calibrator provided to laboratories, the selected strategy was to model the 3 processes described above: immunoenzymatic reaction, metrological traceability and production and routine use conditions.

The model takes into account the physical factors impacting the immuno-enzymatic reaction, the four levels of metrological traceability (Figure 2), and it simulates the distribution of reagent batches in the laboratories.

In order to be reusable, the simulation algorithm also needs to be identical across all the assays available on the Vidas ${ }^{\circledR}$ platform.

\subsection{Analysis of the process}

In order to describe and analyze all the processes to be modeled, different experts were gathered, including R\&D biologists, quality controls experts and biomathematicians. The first step consisted in listing the different steps of each process and the associated variability factors in a chronological order. Then a risk analysis assesses the impact of each factor on the total variability of the process. Based on expert's opinion, the decision was made on the factors to include or not in the model.

\subsection{Statistical method}

The GUM (version 2008) [3] proposes 2 different approaches to estimate uncertainties: the PLU method based on the variances propagation law, and the PDF method (GUM supplement 1 [4]) based on Monte Carlo simulations. Because the conditions to apply the PLU method were not met for immunology assays (nonnormality, non-linearity and high correlation between input factors [5]), the Monte Carlo method was used to develop the simulator.

\subsection{Input data}

During the development of a new assay, designs of experiments are performed to optimize the formulation of the different components of the kits. The results of all these experiments characterize the relationship between the fluorescence signal of the immune-enzymatic reaction and physical factors such as temperature, volumetry, and time. In Figure 3, examples of such relationships are shown.

These results were used as input data for the simulator. 
Figure 3: relationship between signal and physical factors

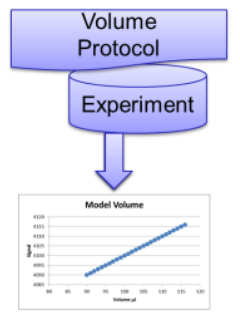

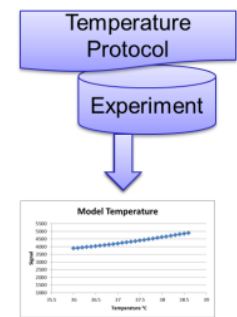

Signal $=f($ temperature

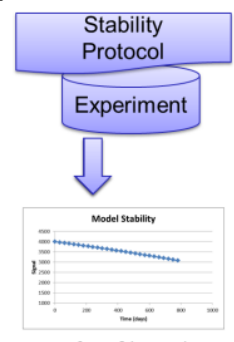

Signal $=f($ Time $)$
Signal $=f($ Volume $)$

Modeling of the metrological traceability process:

International calibrators are used to assign titers to manufacturer's calibrators, which are then used to assign titers to laboratories calibrators (Figure 5).

Thus, three protocols are used (protocol A, B and C), each of them using different calibration curves.

Each protocol consists in testing $n$ calibrators with $m$ replicates (Figure 1). The simulator uses the immunoenzymatic simulation for each of the 3 protocols, to generate the exact number of replicates requested by the protocol ( $n$ calibrators $\mathrm{x} m$ replicates). The complexity of this process is due to the fact that the model needs to take into account the dependency between the titers assigned to the 3 calibrators, and the different numbers of tests of the different calibrators within the 3 protocols.

Figure 5: Traceability and calibration

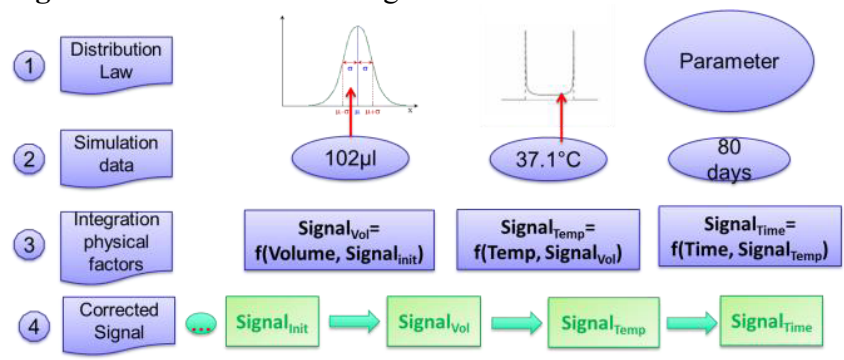

\subsection{Modeling}

Modeling of the immuno-enzymatic reaction:

This model simulates the immune-enzymatic reaction from the raw fluorescence signal. The signal is impacted by different physical factors (Figure 4).

\section{Figure 4: Simulation of the signals}

$1^{\text {st }}$ step: when developing the model, a statistical distribution and parameters are associated to each input factor. For example, normal distribution for the volumetry, arcsine derivative for the temperature.

$2^{\text {nd }}$ step: To perform simulations, a random value for temperature and volume is drawn from each associated distribution. The age of the reagent is considered as a parameter of the simulation.

$3^{\text {rd }}$ step: the simulator then takes into account the relationship between signal and physical factors, established during the development of the product.

$4^{\text {th }}$ step: since the impact of volumetry, temperature and time happens at different moments during the reaction, the signal is modified accordingly in a successive manner. At the end, a simulated signal corresponding to the sample tested is obtained.

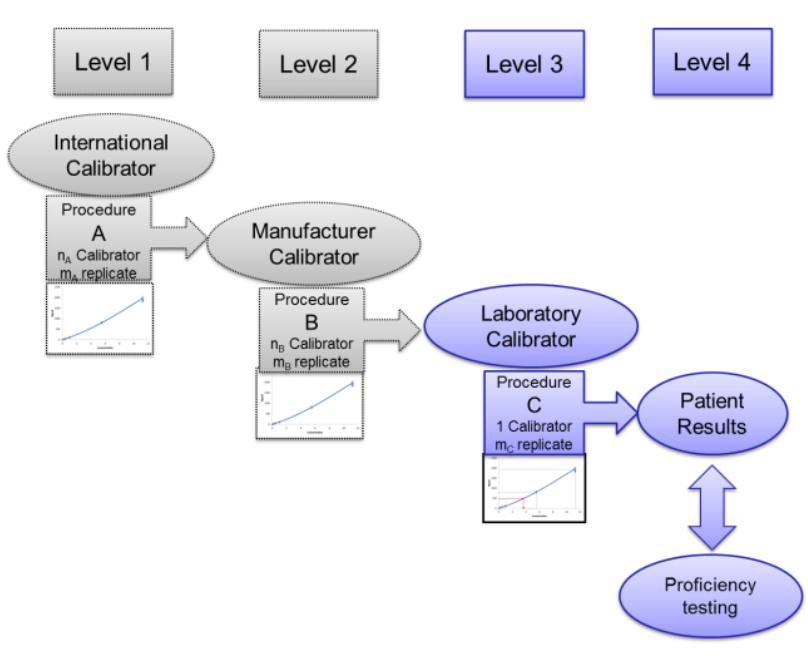

Modeling of the production and routine use process:

The parameters used for this process are the number of reagent batches manufactured per year, the number of customers and the average number of reagents used by the laboratories. Reagents batches will be randomly assigned to laboratories, and each batch affects the uncertainty of each simulated laboratory differently (Figure 6). 
Figure 6: Distribution of the reagents

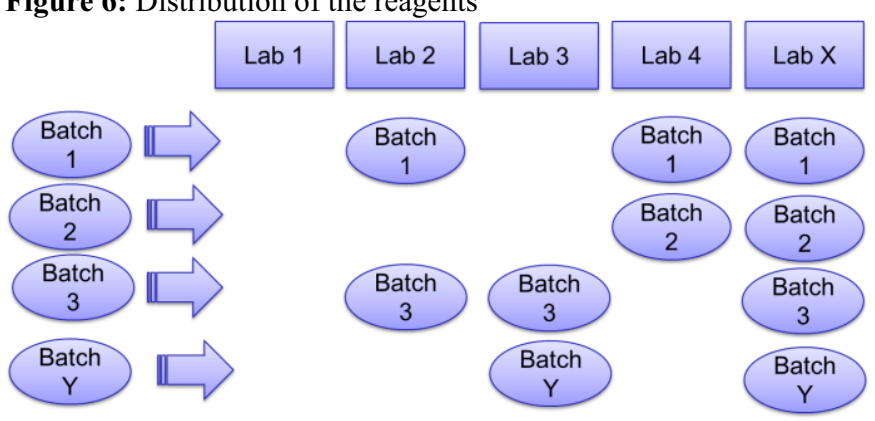

At the end of the validation, around 50 assays passed the validation; thus the model is considered usable for the development of new assay.

\section{Results - Discussion}

\subsection{Results}

The objective of this model was to provide uncertainty associated with the calibrators included in the kits used by the medical laboratories for the 50 or so quantitative assays of the Vidas system. The results were validated using inter-laboratory program results.

An example of simulation results is provided in Figure 7 , showing the acceptable difference compared to the predefined acceptance criteria.

Figure 7: simulated coefficient of variation (green) and interlaboratories coefficient of variation (dark blue) as a function of concentration level.

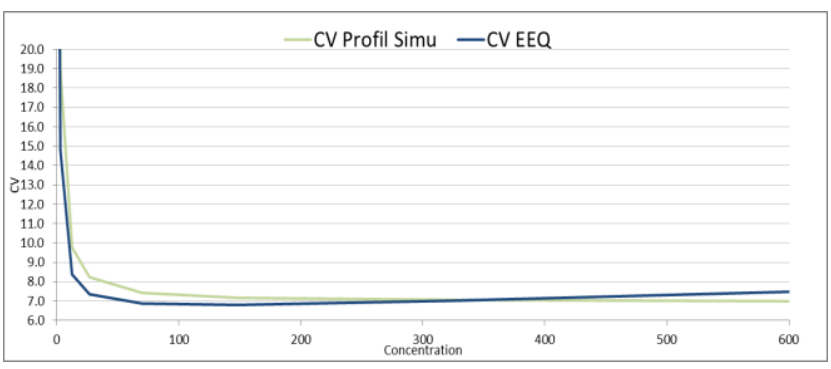

At the end of the simulation, the amount of results obtained by simulation is comparable to the amount of results really obtainable in the field during the lifecycle of the product.

\subsection{Validations}

When using Monte Carlo simulation for uncertainty calculations, three types of validation are needed: validation of the script/software [6], validation of the number of simulations [7] and validation of the uncertainty result itself.

The two first validations mentioned are well standardized and described (eg [6]).

For the validation of the uncertainty result itself, two main risks were identified. The first one is linked to the quality of the input data (are all use conditions well represented? is there some atypical data?). The second one would be to omit some factors in the listing of risk analysis and notation steps, leading to underestimation of the uncertainty. It could also happen that the contribution of a factor could be overestimated because the associated variability could be taken into account both in input data and in the model.

To validate the uncertainty results, outcomes of interlaboratories survey programs (proficiency testing) were taken into account [8]. Comparison between proficiency testing results and simulators results of level 4 (Figure 5) was performed, and validation was considered as passed if the difference remained lower than a predefined acceptance criteria. If the uncertainty is validated for level 4, we can conclude that it is also validated for the manufacturer calibrator (level 3).

\subsection{Perspectives}

In addition to the ultimate uncertainty result, the simulator allows many other applications that could be very helpful to optimize the development process of a new assay. Around forty potential applications have been established, and two of them have already been successfully tested.

For these two cases, the simulator rapidly optimized the quality/cost ratio, reducing the number of concentrations levels needed to establish a calibration curve, while maintaining an adequate quality level. Such optimization by experiments alone would have a required high number of costly experiments, and more time, to obtain the same outcome.

In addition, the accuracy of the results using simulations can be better than the one obtained by experiments. For example, simulations can estimate more sources of variation and thus obtained better predictions.

Simulation is rarely used in In-Vitro Diagnostics companies compared to other industries because the cost of experiments is relatively low. However the cost is increasing more and more because of the development of new technologies and the increased level of regulatory requirements. Simulations are expected to help 
manufacturers to better optimize the number and the conditions of the experiments needed to develop new assays.

The simulator described in this article can be used by different experts through the company (biologists, hardware engineers, and bio mathematicians), and all through the lifecycle of the products, from development of reagents to their commercialization. Thus it is key to gather and validate the knowledge acquired by experiments and through expert's knowledge as input for the simulator.

\section{References}

[1] ISO 17511:2003 - In vitro diagnostic medical devices -- Measurement of quantities in biological samples -Metrological traceability of values assigned to calibrators and control materials.

[2] ISO15189:2012- Laboratoires de biologie médicale - Exigences concernant la qualité et la compétence

[3] JCGM $100: 2008$ "Guide to the expression of uncertainty in measurement" - Introduction

[4] JCGM $101: 2008$ "Guide to the expression of uncertainty in measurement - Propagation of distributions using a Monte Carlo method"

[5] JCGM $101: 2008$ "Guide to the expression of uncertainty in measurement - Propagation of distributions using a Monte Carlo method"- $§ 1$ Scope

[6] IEC 62304:2006(fr) - Logiciels de dispositifs médicaux — Processus du cycle de vie du logiciel- § 5.5/ 5.6

[7] JCGM $101: 2008$ "Guide to the expression of uncertainty in measurement - Propagation of distributions using a Monte Carlo method" $-\S 7.2$ et 7.9

[8] European Federation of National Associations of Measurement, Testing and Analytical Laboratories Technical Report No. 1/2007 - Chapter 1- March 2007 